\title{
Simple and one-pot synthesis of new heterocyclic compounds in three-component reactions between isoquinoline or phenanthridine and acetylenic esters in the presence of $\mathrm{N}$-heterocycles or 1,3-dicarbonyl compounds
}

\author{
Mahmoud Nassiri, ${ }^{b}$ Reza Heydari, ${ }^{a}$ Nourollah Hazeri, ${ }^{a}$ \\ Sayyed Mostafa Habibi-Khorassani, ${ }^{a}$ Malek Taher Maghsoodlou, ${ }^{* a}$ Forough Jalili Milania \\ ${ }^{a}$ Department of Chemistry, The University of Sistan and Baluchestan, P. O. Box 98135-674, \\ Zahedan, Iran \\ ${ }^{b}$ Department of Maritime, The University of Chabahar, Chabahar, Iran \\ E-mail: MT_maghsoodlou@yahoo.com
}

DOI: $\underline{\text { http://dx.doi.org/10.3998/ark.5550190.0011.205 }}$

\begin{abstract}
A new class of enamino esters has been isolated in excellent yields from the 1:1:1 addition reaction between phenanthridine or isoquinoline and acetylenic esters such as ethyl propiolate or dialkyl acetylenedicaboxylates in the presence of heterocyclic $\mathrm{NH}$ compounds (succinimide, indole, 2-methylindole, 2-benzoxazolinone, 6-chlorobenzoxazolinone, carbazole and 3,6dibromocarbazole) or 1,3-dicarbonyl compounds like 1,3-dimethylbarbituric acid, acetylacetone and dibenzoylmethane.
\end{abstract}

Keywords: Enamino esters, phenanthridine, isoquinoline, ethyl propiolate, heterocyclic NH or 1,3-dicarbonyl compounds

\section{Introduction}

The development of simple synthetic routes for widely used organic compounds from readily available reagents is one of the major tasks in organic synthesis. ${ }^{1}$ Phenanthridines are important core structures found in a variety of natural products and other biologically important molecules with a wide range of biological activities and applications, ${ }^{2-10}$ including antibacterial, antiprotozoal, anticancer, antimicrobial, anti-inflammatory, antivirial, antioxidant ${ }^{11-21}$ and also with applications as drugs, ${ }^{8}$ DNA targeting agents, ${ }^{22}$ dyes, ${ }^{23}$ and probes. ${ }^{24}$ Isoquinoline is also present in various natural products such as cryptaustoline and cryptowoline. ${ }^{25}$ They are known to exhibit various biological activities ${ }^{26-34}$ such as antileukaemic, ${ }^{35}$ tubulin polymerization inhibitory $^{36}$ and anti-tumour activities. ${ }^{37}$ As previously reported, ${ }^{38}$ reaction between 
phenanthridine and two mol equivalents of dimethyl acetylenedicarboxylate, leads to the formation of a new ring fused to the phenanthridine. In the current work, we now describe an efficient synthesis of a new class of enamino esters (see Scheme 1).

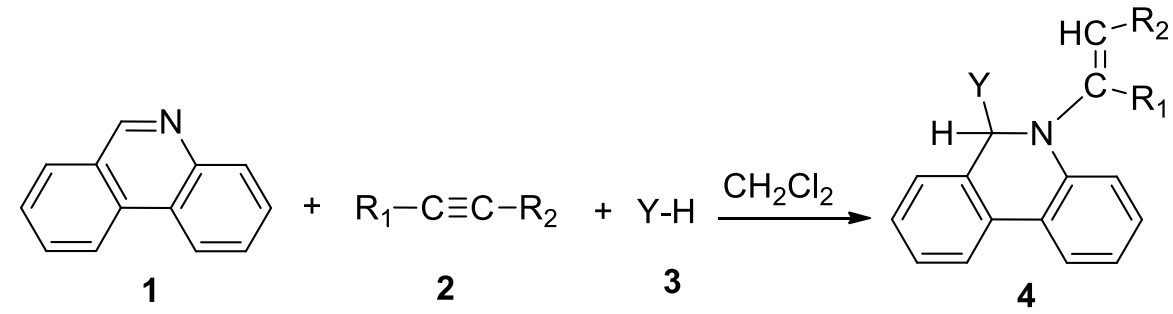

4(a-c): $\mathrm{R}_{1}=\mathrm{H}, \mathrm{R}_{2}=\mathrm{CO}_{2} \mathrm{Et} \quad 4(\mathrm{~d}, \mathrm{e}): \mathrm{R}_{1}=\mathrm{R}_{2}=\mathrm{CO}_{2} \mathrm{Me}$

$4(\mathrm{f}, \mathrm{g}): \mathrm{R}_{1}=\mathrm{R}_{2}=\mathrm{CO}_{2} \mathrm{Et} \quad 4 \mathrm{~h}: \mathrm{R}_{1}=\mathrm{R}_{2}=\mathrm{CO}_{2} \mathrm{Bu}^{\mathrm{t}}$

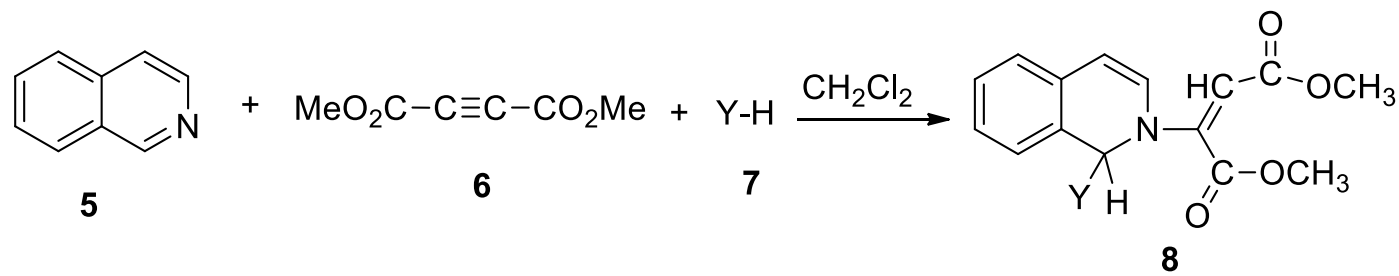

\begin{tabular}{|c|c|c|c|c|c|c|c|}
\hline & $\mathrm{Y}$ & $\mathrm{Z}$ or $\mathrm{E}$ & $\%$ Yeld & & $\mathrm{Y}$ & $\mathrm{Z}$ or $\mathrm{E}$ & $\%$ Yeld \\
\hline $4 a$ & & $\mathrm{E}$ & 92 & $4 \mathrm{f}$ & & $\mathrm{E}$ & 94 \\
\hline $4 b$ & & $\mathrm{E}$ & 93 & $4 \mathrm{j}$ & & $\mathrm{E}$ & 95 \\
\hline $4 c$ & & $\mathrm{E}$ & 92 & $4 h$ & & $\mathrm{E}$ & 96 \\
\hline $4 d$ & & $\mathrm{E}$ & 94 & $4 \mathrm{i}$ & & $\mathrm{Z}$ & 93 \\
\hline $4 e$ & & $\mathrm{E}$ & 92 & $4 j$ & & $\mathrm{Z}$ & 91 \\
\hline
\end{tabular}

Scheme 1 


\section{Results and Discussion}

An efficient synthesis of a new class of enamino esters from reaction between phenanthridine $\mathbf{1}$ or isoquinoline $\mathbf{5}$ and activated acetylenic esters $\mathbf{2}$ or $\mathbf{6}$ as a Michael acceptor ${ }^{39-46}$ was undertaken in the presence of heterocyclic NH compounds (succinimide, indole and 2-methylindole, 2benzoxazolinone, 6-chlorobenzoxazolinone, carbazole and 3,6-dibromocarbazole) or 1,3dicarbonyl compounds such as 1,3-dimethylbarbituric acid, acetylacetone and dibenzoylmethane, at ambient temperature. Reactions were carried out by first mixing the phenanthridine or isoquinoline and heterocyclic $\mathrm{NH}$ or 1,3-dicarbonyl compounds and then the acetylenic ester was added slowly. The reactions proceeded smoothly in $\mathrm{CH}_{2} \mathrm{Cl}_{2}$ and then the whole reaction mixture solidified into yellow solid within a few hours. The ${ }^{1} \mathrm{H}$ and ${ }^{13} \mathrm{C}$ NMR spectra of the crude products clearly indicated the formation of enamino esters $\mathbf{4 a - h}$ and $\mathbf{8 i}, \mathbf{j}$. No product other than $\mathbf{4 a - h}$ and $\mathbf{8 i}, \mathbf{j}$ could be detected by NMR spectroscopy. The structures of compounds $\mathbf{4 a - h}$ and 8i,j were confirmed by elemental analyses, mass, IR, ${ }^{1} \mathrm{H}$ NMR and ${ }^{13} \mathrm{C}$ NMR spectra. The ${ }^{1} \mathrm{H}$ NMR spectrum of $\mathbf{4 a}$ exhibited signals for the methyl $\left(\delta=1.29,3 \mathrm{H}, \mathrm{t},{ }^{3} J_{\mathrm{HH}}=7.2 \mathrm{~Hz}\right.$, $\left.\mathrm{OCH}_{2} \mathrm{CH}_{3}\right)$, methylene $\left(\delta=4.19,2 \mathrm{H}, \mathrm{q},{ }^{3} \mathrm{~J}_{\mathrm{HH}}=7.2 \mathrm{~Hz}, \mathrm{OCH}_{2} \mathrm{CH}_{3}\right)$, and olefinic $(\delta=5.58$ and 8.36, 2d, ${ }^{3} J_{\mathrm{HH}}=13.6 \mathrm{~Hz}, \mathrm{CH}=\mathrm{CH}-\mathrm{OCH}_{2} \mathrm{CH}_{3}$ ) protons, along with multiplets at $\delta=7.13-8.09$ ppm for the aromatic protons. The NCHN moieties at $\delta=9.03-9.33 \mathrm{ppm}$ in compounds 4 a-e are deshielded due to the anisotropic effect of a benzene ring of phenanthridine. ${ }^{49}$ The ${ }^{13} \mathrm{C}$ NMR spectrum of $4 \mathbf{a}$ showed 22 distinct resonances in agreement with the proposed structure. In addition, product $4 \mathbf{a}$ displayed ${ }^{13} \mathrm{C}$ NMR resonances at $\delta 94.06 \mathrm{ppm}, 115.84$ and $120.72 \mathrm{ppm}$, respectively for the $\mathrm{NCHN}, \mathrm{N}-\mathrm{CH}=\mathrm{CH}-\mathrm{CO}_{2} \mathrm{CH}_{2} \mathrm{CH}_{3}$, and $\mathrm{N}-\mathrm{CH}=\mathrm{CH}-\mathrm{CO}_{2} \mathrm{CH}_{2} \mathrm{CH}_{3}$ units. The carbonyl group resonances in the ${ }^{13} \mathrm{C}$ NMR spectra of $\mathbf{4 a}$ appeared at $\delta=174.78$ and 177.16 ppm. The ${ }^{1} \mathrm{H}$ and ${ }^{13} \mathrm{C}$ NMR spectra of compounds $\mathbf{4 b}-\mathbf{h}$ and $\mathbf{8 i}, \mathbf{j}$ are similar to those of $\mathbf{4 a}$. Assignment of the configuration ( $Z$ or $E$ ) in compounds $\mathbf{4 a - h}$ and $\mathbf{8 i} \mathbf{j} \mathbf{j}$ was decided on the basis of the chemical shift of the olefinic proton. ${ }^{34,47-48}$ With respect to the same employed conditions (effect of same solvent and temperature in our reactions) it seems that, the structural effect of reactants is an important factor for assignment of the configuration ( $Z$ or $E$ ) (see Scheme 2).

Briefly, we have developed a new method to access a novel class of heterocyclic derivatives. The present method has the advantage that, not only is the reaction performed under neutral conditions, but also the substances can be mixed without any activation or modifications. It seems that, this procedure is easy and simple approach for synthesis of heterocyclic derivatives. 

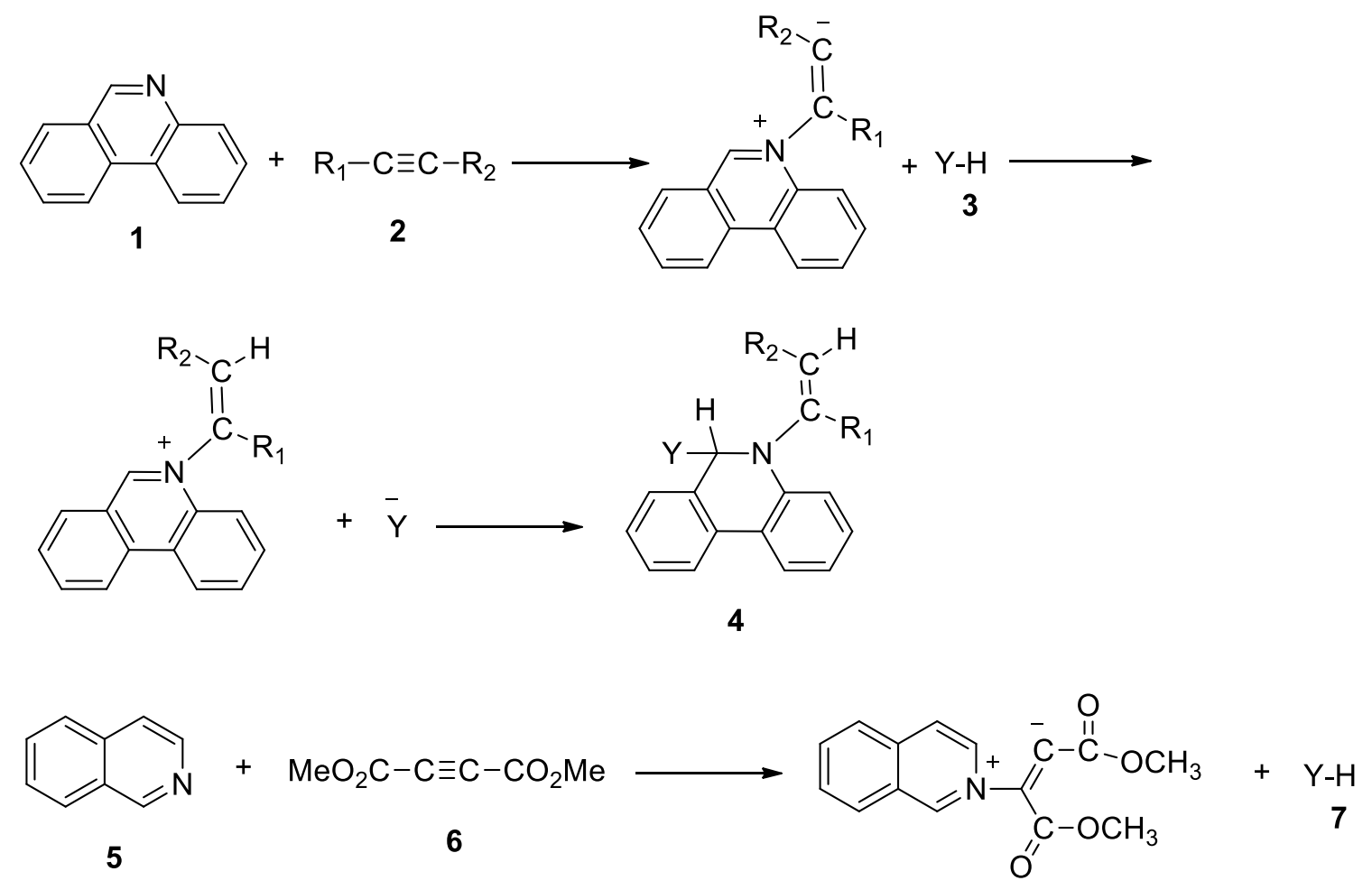<smiles>[Y]C1([Y])c2ccccc2C=CN1/C(=C/C(=O)OC)C(=O)OC</smiles>

\section{Scheme 2}

\section{Experimental Section}

General. Melting points and IR spectra of all compounds were measured on an Electrothermal 9100 apparatus and a Shimadzu IR-460 spectrometer, respectively. The ${ }^{1} \mathrm{H},{ }^{13} \mathrm{C}$, and ${ }^{31} \mathrm{P}$ NMR spectra were obtained with a BRUKER DRX-500 AVANCE instrument using $\mathrm{CDCl}_{3}$ as applied solvent and TMS as internal standard at 500.1, 125.8, and 202.4 MHz respectively. In addition, the mass spectra were recorded on a GCMS-QP5050A mass spectrometer operating at an ionization potential of $70 \mathrm{eV}$. Elemental analysis for $\mathrm{C}, \mathrm{H}$ and $\mathrm{N}$ were performed using a Heraeus $\mathrm{CHN}-\mathrm{O}-\mathrm{Rapid}$ analyzer. Activated acetylenic esters, phenanthridine, isoquinoline, succinimide, indole, 2-methylindole, 2-benzoxazolinone, 6-chloro- benzoxazolinone, carbazole, 3,6dibromocarbazole, 1,3-dimethylbarbituric acid, acetylacetone and dibenzoylmethane were purchased from Fluka, (Buchs, Switzerland) and used without further purification. 
General synthetic procedure, exemplified by (E)-ethyl 3-(6-(2,5-dioxopyrrolidin-1yl)phenanthridine-5(6H)-yl)acrylate 4a. To a magnetically stirred solution of phenanthridine (0.18 g, $1 \mathrm{mmol})$ and succinimide $(0.09 \mathrm{~g}, 1 \mathrm{mmol})$ in $\mathrm{CH}_{2} \mathrm{Cl}_{2}(10 \mathrm{~mL})$ was added, dropwise, a mixture of ethyl propiolate $(1 \mathrm{mmol})$ in $\mathrm{CH}_{2} \mathrm{Cl}_{2}(5 \mathrm{~mL})$ at $-10{ }^{\circ} \mathrm{C}$ over $10 \mathrm{~min}$. After a few hours stirring at ambient temperature, the whole reaction mixture solidified into a brown solid, the solvent was then removed under reduced pressure and product washed with cold diethyl ether $(2 \times 5 \mathrm{~mL})$. Then the product was recrystallized from a mixture of acetonitrile and acetone.

Brown powder, yield 92\%, $0.35 \mathrm{~g} \mathrm{mp} \mathrm{100-102}{ }^{\circ} \mathrm{C}$, IR $\left(v_{\max }, \mathrm{cm}^{-1}\right): 1707$ and $1773(\mathrm{C}=\mathrm{O}) .{ }^{1} \mathrm{H}$ $\operatorname{NMR}\left(500.1 \mathrm{MHz}, \mathrm{CDCl}_{3}\right), \delta_{\mathrm{H}} 1.29\left(3 \mathrm{H}, \mathrm{t},{ }^{3} J_{\mathrm{HH}}=7.2 \mathrm{~Hz}, \mathrm{OCH}_{2} \mathrm{CH}_{3}\right), 2.75\left(4 \mathrm{H}, \mathrm{s}, 2 \mathrm{CH}_{2}\right), 4.19$ $\left(2 \mathrm{H}, \mathrm{q},{ }^{3} \mathrm{~J}_{\mathrm{HH}}=7.2 \mathrm{~Hz}, \mathrm{OCH}_{2} \mathrm{CH}_{3}\right), 5.85\left(1 \mathrm{H}, \mathrm{d},{ }^{3} \mathrm{~J}_{\mathrm{HH}}=13.6 \mathrm{~Hz}, \mathrm{~N}-\mathrm{CH}=\mathrm{CH}-\mathrm{CO}_{2} \mathrm{CH}_{2} \mathrm{CH}_{3}\right), 7.13-$ $8.09\left(8 \mathrm{H}_{\text {aro }}, \mathrm{m}, 8 \mathrm{CH}\right.$ phenanthridine $), 8.60\left(1 \mathrm{H}, \mathrm{d},{ }^{3} \mathrm{~J}_{\mathrm{HH}}=13.6 \mathrm{~Hz}, \mathrm{~N}-\mathrm{CH}=\mathrm{CH}-\mathrm{CO}_{2} \mathrm{CH}_{2} \mathrm{CH}_{3}\right)$, $9.03(1 \mathrm{H}, \mathrm{s}, \mathrm{NCHN}) .{ }^{13} \mathrm{C} \mathrm{NMR}\left(125.8 \mathrm{MHz}, \mathrm{CDCl}_{3}\right), \delta_{\mathrm{C}} 13.38\left(\mathrm{OCH}_{2} \mathrm{CH}_{3}\right), 26.87\left(\mathrm{OCH}_{2} \mathrm{CH}_{3}\right)$, $28.51\left(2 \mathrm{CH}_{2}\right), 94.06(\mathrm{NCHN}), 115.84\left(1 \mathrm{C}, \mathrm{N}-\mathrm{CH}=\mathrm{CH}-\mathrm{CO}_{2} \mathrm{CH}_{2} \mathrm{CH}_{3}\right), 120.72(1 \mathrm{C}, \mathrm{N}-\mathrm{CH}=\mathrm{CH}-$ $\left.\mathrm{CO}_{2} \mathrm{CH}_{2} \mathrm{CH}_{3}\right), 121.09,122.06,122.53,125.68,126.05,126.41,126.92,127.67,127.78,128.65$, 130.05 and 152.24 (12C, phenanthridine), $174.78(\mathrm{C}=\mathrm{O}$, ester), 177.16 (2C=O, succinimide). Anal. Calcd for $\mathrm{C}_{22} \mathrm{H}_{20} \mathrm{~N}_{2} \mathrm{O}_{4}$ (376.16): C, 70.18; H, 5.36; N, 7.44\%, Found: C, 70.21; H, 5.31; N, $7.60 \%$.

Ethyl $(E)-3-(6-(1 H$-indole-1-yl)phenanthridine-5(6H)-yl)acrylate 4b. Yellow powder, yield 93\%, $0.37 \mathrm{~g} \mathrm{mp} \mathrm{138-140}{ }^{\circ} \mathrm{C}$, IR $\left(v_{\max }, \mathrm{cm}^{-1}\right)$ : $1718(\mathrm{C}=\mathrm{O}) .{ }^{1} \mathrm{H}$ NMR $\left(500.1 \mathrm{MHz}, \mathrm{CDCl}_{3}\right), \delta_{\mathrm{H}}$ $1.18\left(3 \mathrm{H}, \mathrm{t},{ }^{3} \mathrm{~J}_{\mathrm{HH}}=7.1 \mathrm{~Hz}, \mathrm{OCH}_{2} \mathrm{CH}_{3}\right), 4.19\left(2 \mathrm{H}, \mathrm{q},{ }^{3} \mathrm{~J}_{\mathrm{HH}}=7.1 \mathrm{HZ}, \mathrm{OCH}_{2} \mathrm{CH}_{3}\right), 5.45\left(1 \mathrm{H}, \mathrm{d},{ }^{3} J_{\mathrm{HH}}\right.$ $\left.=16.6 \mathrm{HZ}, \mathrm{N}-\mathrm{CH}=\mathrm{CH}-\mathrm{CO}_{2} \mathrm{CH}_{2} \mathrm{CH}_{3}\right), 6.25\left(1 \mathrm{H}, \mathrm{d},{ }^{3} J_{\mathrm{HH}}=3.5 \mathrm{~Hz}, \mathrm{~N}-\mathrm{CH}=\mathrm{CH}\right.$, indole $), 6.31(1 \mathrm{H}$, $\mathrm{d},{ }^{3} \mathrm{~J}_{\mathrm{HH}} 3.5 \mathrm{~Hz}, \mathrm{~N}-\mathrm{CH}=\mathrm{CH}$, indole $), 7.14-8.03\left(12 \mathrm{H}_{\text {arom }}, \mathrm{m}, 12 \mathrm{CH}\right), 8.30\left(1 \mathrm{H}, \mathrm{d},{ }^{3} \mathrm{~J}_{\mathrm{HH}}=16.6 \mathrm{~Hz}\right.$, $\left.\mathrm{N}-\mathrm{CH}=\mathrm{CH}-\mathrm{CO}_{2} \mathrm{CH}_{2} \mathrm{CH}_{3}\right), 9.24(1 \mathrm{H}, \mathrm{s}, \mathrm{NCHN}) .{ }^{13} \mathrm{C} \mathrm{NMR}\left(125.8 \mathrm{MHz}, \mathrm{CDCl}_{3}\right), \delta_{\mathrm{C}} 13.43$ $\left(\mathrm{OCH}_{2} \mathrm{CH}_{3}\right), 25.71\left(\mathrm{OCH}_{2} \mathrm{CH}_{3}\right), 63.76(\mathrm{NCHN}), 96.41\left(\mathrm{C}, \mathrm{N}-\mathrm{CH}=\mathrm{CH}-\mathrm{CO}_{2} \mathrm{CH}_{2} \mathrm{CH}_{3}\right), 111.25$, 113.30, 120.19 and 120.29 (4C, indole), 121.85, 122.56 and 122.53 (3C, phenanthridine), 123.26 and 124.30 (2C, indole), 125.66 (1C, $\left.\mathrm{N}-\mathrm{CH}=\mathrm{CH}-\mathrm{CO}_{2} \mathrm{CH}_{2} \mathrm{CH}_{3}\right), \quad 125.68,126.35$ 126.93,127.29, 127.78, 129.65 and 130.15 (7C, phenanthridine), 137.14 (1C, indole), 137.43 and 139.18 (2C, phenanthridine), 153.09 (1C, indole), 172.34 (C=O, ester). MS, m/z (\%) = 364 (MEt and $\mathrm{H}, 13), 278\left(\mathrm{C}_{18} \mathrm{H}_{16} \mathrm{NO}_{2}, 100\right), 204\left(\mathrm{M}-\mathrm{C}_{8} \mathrm{H}_{6} \mathrm{~N}, \mathrm{CO}_{2} \mathrm{Et}\right.$ and $\left.\mathrm{H}, 48\right), 179\left(\mathrm{C}_{13} \mathrm{H} 9 \mathrm{~N}, 60\right), 97$ $\left(\mathrm{C}_{5} \mathrm{H}_{5} \mathrm{O}_{2}\right.$, 26). Anal. Calcd for $\mathrm{C}_{26} \mathrm{H}_{22} \mathrm{~N}_{2} \mathrm{O}_{2}$ (394.17): C, 79.15; H, 5.62; N, 7.10\%, Found: C, 78.98; H, 5.64; N, 7.18\%.

Ethyl (E)-3-(6-(2-methyl-1H-indole-1-yl)phenanthridine-5(6H)-yl)acrylate 4c. Brown powder, yield $92 \%, 0.38 \mathrm{~g}$; mp 136-138 ${ }^{\circ} \mathrm{C}$, IR $\left(v_{\max }, \mathrm{cm}^{-1}\right): 1723(\mathrm{C}=\mathrm{O}) .{ }^{1} \mathrm{H}$ NMR $(500.1 \mathrm{MHz}$, $\left.\mathrm{CDCl}_{3}\right), \delta \mathrm{H} 1.25\left(3 \mathrm{H}, \mathrm{t},{ }^{3} \mathrm{~J}_{\mathrm{HH}}=7.2 \mathrm{~Hz}, \mathrm{OCH}_{2} \mathrm{CH}_{3}\right), 2.39\left(3 \mathrm{H}, \mathrm{s}, \mathrm{CH}_{3}\right), 4.18\left(2 \mathrm{H}, \mathrm{q},{ }^{3} J_{\mathrm{HH}}=7.2\right.$ $\left.\mathrm{Hz}, \mathrm{OCH}_{2} \mathrm{CH}_{3}\right), 5.40\left(1 \mathrm{H}, \mathrm{d},{ }^{3} \mathrm{~J}_{\mathrm{HH}}=13.3 \mathrm{~Hz}, \mathrm{~N}-\mathrm{CH}=\mathrm{CH}-\mathrm{CO}_{2} \mathrm{CH}_{2} \mathrm{CH}_{3}\right), 6.22(1 \mathrm{H}$, s, indole), 7.08-8.63 $\left(12 \mathrm{H}_{\text {aro }}, \mathrm{m}, 12 \mathrm{CH}\right), 8.70\left(1 \mathrm{H}, \mathrm{d},{ }^{3} \mathrm{~J}_{\mathrm{HH}}=13.3 \mathrm{~Hz}, \mathrm{~N}-\mathrm{CH}=\mathrm{CH}-\mathrm{CO}_{2} \mathrm{CH}_{2} \mathrm{CH}_{3}\right), 9.30(1 \mathrm{H}$, $\mathrm{NCHN}) .{ }^{13} \mathrm{C} \mathrm{NMR}\left(125.8 \mathrm{MHz}, \mathrm{CDCl}_{3}\right), \delta \mathrm{C} 14.03\left(\mathrm{OCH}_{2} \mathrm{CH}_{3}\right), 26.80\left(\mathrm{OCH}_{2} \mathrm{CH}_{3}\right), 59.41$ $(\mathrm{NCHN}), 97.66\left(1 \mathrm{C}, \mathrm{N}-\mathrm{CH}=\mathrm{CH}-\mathrm{CO}_{2} \mathrm{CH}_{2} \mathrm{CH}_{3}\right), 110.18,113.14,119.12,119.75122 .16$ and 123.30 (6C, indole), 123.80 and 125.95 (2C, phenanthridine), $126.03\left(\mathrm{~N}-\mathrm{CH}=\mathrm{CH}-\mathrm{CO}_{2} \mathrm{CH}_{2} \mathrm{CH}_{3}\right)$, 126.81, 126.93, 127.47, 127.70, 129.92 and 131.11 (8C, phenanthridine), 135.18 (1C, indole), 
137.23 and 138.10 (2C, phenanthridine), 152.43 (1C, indole), 174.64 (C=O, ester). $\mathrm{MS}, \mathrm{m} / \mathrm{z}(\%)$ $=408\left(\mathrm{M}^{+}, 3\right), 379\left(\mathrm{M}-\mathrm{CH}_{2} \mathrm{CH}_{3}, 7\right), 335\left(\mathrm{M}-\mathrm{CO}_{2} \mathrm{CH}_{2} \mathrm{CH}_{3}, 39\right), 179\left(\mathrm{C}_{13} \mathrm{H}_{9} \mathrm{~N}, 37\right), 130\left(\mathrm{C}_{9} \mathrm{H}_{8} \mathrm{~N}\right.$, 100). Anal. Calcd for $\mathrm{C}_{27} \mathrm{H}_{24} \mathrm{~N}_{2} \mathrm{O}_{2}$ (408.19): C, 79.37; H, 5.93; N, $6.86 \%$, Found: C, 79.42; H, 6.02; N, $6.91 \%$.

Dimethyl 2-(6-(2-oxobenzo[d]oxazol-3(2H)-yl)phenanthridine-5(6H)-yl)fumarate 4d. Yellow powder, yield 94\%, $0.43 \mathrm{~g} \mathrm{mp} \mathrm{112-114}{ }^{\circ} \mathrm{C}$, IR $\left(v_{\max }, \mathrm{cm}^{-1}\right): 1722$ and $1783(\mathrm{C}=\mathrm{O}) .{ }^{1} \mathrm{H}$ NMR $\left(500.1 \mathrm{MHz}, \mathrm{CDCl}_{3}\right), \delta \mathrm{H} 3.71$ and $3.88\left(6 \mathrm{H}, 2 \mathrm{~s}, 2 \mathrm{OCH}_{3}\right), 7.08\left(1 \mathrm{H}, \mathrm{s}, \mathrm{N}-\mathrm{C}=\mathrm{CH}-\mathrm{CO}_{2} \mathrm{CH}_{3}\right), 7.12-$ $8.63\left(12 \mathrm{H}_{\text {arom }}, \mathrm{m}, 12 \mathrm{CH}\right), 9.35(1 \mathrm{H}, \mathrm{s}, \mathrm{NCHN}) .{ }^{13} \mathrm{C} \mathrm{NMR}\left(125.8 \mathrm{MHz}, \mathrm{CDCl}_{3}\right), \delta_{\mathrm{C}} 52.69$ and $53.70\left(2 \mathrm{OCH}_{3}\right), 109.62(\mathrm{NCHN}), 110.41\left(\mathrm{~N}-\mathrm{C}=\mathrm{CH}-\mathrm{CO}_{2} \mathrm{CH}_{3}\right), 109.07,112.98$ and $121.71(3 \mathrm{C}$, benzoxazole), 121.91, 122.33 and 123.40 (3C, phenanthridine), 123.82 (1C, benzoxazole), $124.06\left(\mathrm{~N}-\mathrm{C}=\mathrm{CH}-\mathrm{CO}_{2} \mathrm{CH}_{3}\right), \quad 124.17, \quad 125.96, \quad 127.79, \quad 128.94,129.00$ and $129.19(6 \mathrm{C}$, phenanthridine), 142.23 (1C, benzoxazole), 154.32 ( $1 \mathrm{C}$, benzoxazole, $\mathrm{N}-\mathrm{C}=\mathrm{O}$ ), 165.96 and 162.73 (2C=O, ester). Anal. Calcd for $\mathrm{C}_{26} \mathrm{H}_{20} \mathrm{~N}_{2} \mathrm{O}_{6}$ (456.16): C, 68.40; H, 4.42; N, 6.14\%, Found: C, 68.49; H, 4.50; N, 6.23\%.

Dimethyl 2-(6-(6-chloro-2-oxobenzo[d]oxazol-3(2H)-yl)phenanthridine-5(6H(-yl)fumarate

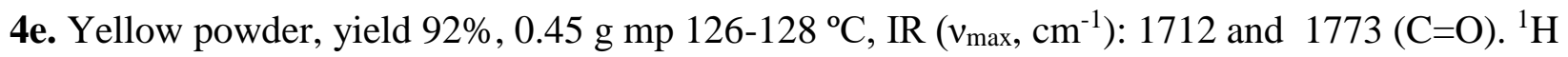
NMR (500.1 MHz, $\left.\mathrm{CDCl}_{3}\right), \delta_{\mathrm{H}} 3.69$ and $3.85\left(6 \mathrm{H}, 2 \mathrm{~s}, 2 \mathrm{OCH}_{3}\right), 6.65\left(1 \mathrm{H}, \mathrm{s}, \mathrm{N}-\mathrm{C}=\mathrm{CH}-\mathrm{CO}_{2} \mathrm{CH}_{3}\right)$, 7.09-8.55 $\left(11 \mathrm{H}_{\text {aro }}, \mathrm{m}, 11 \mathrm{CH}\right), 9.33(1 \mathrm{H}, \mathrm{s}, \mathrm{NCHN}) .{ }^{13} \mathrm{C} \mathrm{NMR}\left(125.8 \mathrm{MHz}, \mathrm{CDCl}_{3}\right) \delta_{\mathrm{C}} 52.48$ and $53.50\left(2 \mathrm{OCH}_{3}\right), 76.69(\mathrm{NCHN}), 110.10\left(\mathrm{~N}-\mathrm{C}=\mathrm{CH}-\mathrm{CO}_{2} \mathrm{CH}_{3}\right), 110.95,114.23$ and $122.70(3 \mathrm{C}$, benzoxazole), 122.91, 122.86 and 124.24 (3C, phenanthridine), 123.06 (1C, benzoxazole), $123.92\left(\mathrm{~N}-\mathrm{C}=\mathrm{CH}-\mathrm{CO}_{2} \mathrm{CH}_{3}\right), 125.47,127.42,127.66,128.13,128.45,128.92,129.14$ and 129.25 (7C, phenanthridine), 131.67 (1C, benzoxazole), 132.20 and 132.61 (2C, phenanthridine), 142.75 (1C, benzoxazole), 152.38 ( $1 \mathrm{C}$, benzoxazole, $\mathrm{N}-\mathrm{C}=\mathrm{O}), 161.43$ and 162.33 (2C=O, ester). Anal. Calcd for $\mathrm{C}_{26} \mathrm{H}_{19} \mathrm{ClN}_{2} \mathrm{O}_{6}$ (490.65): C, 63.59; H, 3.90; N, 5.71\%, Found: C, 63.67; H, 3.98; N, $5.81 \%$.

Diethyl 2-(6-(9H-carbazole-9-yl)phenanthridine-5(6H)-yl)fumarate 4f. Yellow powder, yield 94\%, $0.49 \mathrm{~g} \mathrm{mp} \mathrm{121-123}{ }^{\circ} \mathrm{C}$, IR $\left(v_{\max }, \mathrm{cm}^{-1}\right)$ : 1716 and $1756(\mathrm{C}=\mathrm{O}) .{ }^{1} \mathrm{H}$ NMR $(500.1 \mathrm{MHz}$, $\left.\mathrm{CDCl}_{3}\right), \delta_{\mathrm{H}} 0.75$ and $0.98\left(6 \mathrm{H}, 2 \mathrm{t},{ }^{3} \mathrm{~J}_{\mathrm{HH}}=6.9 \mathrm{~Hz}, 2 \mathrm{OCH}_{2} \mathrm{CH}_{3}\right), 3.75$ and $3.86(4 \mathrm{H}, 2 \mathrm{~m}, 2 \mathrm{ABX} 3$ system, 2OCH $\left.\mathrm{CH}_{3}\right), 6.30\left(1 \mathrm{H}, \mathrm{s}, \mathrm{N}-\mathrm{C}=\mathrm{CH}-\mathrm{CO}_{2} \mathrm{CH}_{2} \mathrm{CH}_{3}\right), 7.28(1 \mathrm{H}, \mathrm{s}, \mathrm{NCHN}), 7.04-8.07$ $\left(16 \mathrm{H}_{\text {arom }}, \mathrm{m}, 16 \mathrm{CH}\right) .{ }^{13} \mathrm{C} \mathrm{NMR}\left(125.8 \mathrm{MHz}, \mathrm{CDCl}_{3}\right) \delta_{\mathrm{C}} 14.11$ and $14.84\left(2 \mathrm{OCH}_{2} \mathrm{CH}_{3}\right), 59.12$ and $61.51\left(2 \mathrm{OCH}_{2} \mathrm{CH}_{3}\right), 69.18(\mathrm{NCHN}), 107.72\left(1 \mathrm{C}, \mathrm{N}-\mathrm{C}=\mathrm{CH}-\mathrm{CO}_{2} \mathrm{CH}_{2} \mathrm{CH}_{3}\right), 114.75,119.35$, 121.18 and 121.89 (4C, phenanthridine), 122.07 and 122.69 (2C, carbazole), 124.84 (1C, N$\mathrm{C}=\mathrm{CH}-\mathrm{CO}_{2} \mathrm{CH}_{2} \mathrm{CH}_{3}$ ), 125.06 and 126.63 (2C, phenanthridine), 126.39 (1C, carbazole), 127.11, 129.28, 130.17, 131.40 and 132.17 (5C, phenanthridine), 134.56, 135.46, 136.20, 137.12, 138.71, 139.53, 139.60, 139.94 and 142.07 (9C, carbazole), 142.01 (1C, phenanthridine), 164.19 and $165.14(2 \mathrm{C}=\mathrm{O}$, ester). $\mathrm{MS}, \mathrm{m} / \mathrm{z}(\%)=350$ (M-carbazole, 100), 204 (M-carbazole and $\left.2 \mathrm{CO}_{2} \mathrm{Et}, 21\right), 179\left(\mathrm{C}_{13} \mathrm{H}_{9} \mathrm{~N}, 38\right)$. Anal. Calcd for $\mathrm{C}_{33} \mathrm{H}_{28} \mathrm{~N}_{2} \mathrm{O}_{4}$ (516.22): C, 76.71; H, 5.47; N, $5.42 \%$, Found: C, 76.80; H, 5.52; N, 5.36\%.

Diethyl 2-(6-(3,6-diboromo-9H-carbazole-9-yl)phenanthridine-5(6H)-yl)fumarate 4g. Yellow

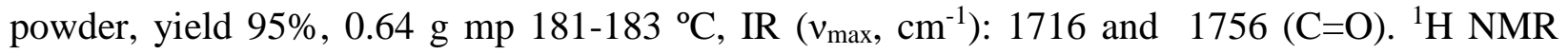


$\left(500.1 \mathrm{MHz}, \mathrm{CDCl}_{3}\right), \delta_{\mathrm{H}} 0.82$ and $1.03\left(6 \mathrm{H}, 2 \mathrm{t},{ }^{3} \mathrm{~J}_{\mathrm{HH}}=7.1 \mathrm{~Hz}, 2 \mathrm{OCH}_{2} \mathrm{CH}_{3}\right), 3.56$ and $3.84(4 \mathrm{H}$, 2m, 2ABX3system, 2OCH $\left.\mathrm{CH}_{3}\right), 6.38\left(1 \mathrm{H}, \mathrm{s}, \mathrm{N}-\mathrm{C}=\mathrm{CH}-\mathrm{CO}_{2} \mathrm{CH}_{2} \mathrm{CH}_{3}\right), 7.99-8.10\left(14 \mathrm{H}_{\text {aro }}, \mathrm{m}\right.$, $14 \mathrm{CH}), 8.13(1 \mathrm{H}, \mathrm{s}, \mathrm{NCHN}) .{ }^{13} \mathrm{C}$ NMR $\left(125.8 \mathrm{MHz}, \mathrm{CDCl}_{3}\right), 13.53$ and $13.74\left(2 \mathrm{OCH}_{2} \mathrm{CH}_{3}\right)$, 60.96 and $61.91\left(2 \mathrm{OCH}_{2} \mathrm{CH}_{3}\right), 69.53(\mathrm{NCHN}), 112.23\left(1 \mathrm{C}, \mathrm{N}-\mathrm{C}=\mathrm{CH}-\mathrm{CO}_{2} \mathrm{CH}_{2} \mathrm{CH}_{3}\right), 113.44$, 115.73, 121.19 and 122.59 (5C, phenanthridine), 122.94, 123.09, 123.30, 123.69 and 123.86 (5C, carbazole), $124.41\left(1 \mathrm{C}, \mathrm{N}-\mathrm{C}=\mathrm{CH}-\mathrm{CO}_{2} \mathrm{CH}_{2} \mathrm{CH}_{3}\right), 124.70,125.25,125.30$ and $127.82 \quad(4 \mathrm{C}$, phenanthridine), 129.08, 129.18 and 129.32 (3C, carbazole), 130.38, 130.56 and 131.41 (3C, phenanthridine), 138.41, 140.90, 140.99 and 141.49 (4C, carbazole), 163.30 and $163.41(2 \mathrm{C}=\mathrm{O}$, ester). Anal. Calcd for $\mathrm{C}_{33} \mathrm{H}_{26} \mathrm{Br}_{2} \mathrm{~N}_{2} \mathrm{O}_{4}$ (674.20): C, 58.74; H, 3.89; N, 4.15\%, Found: C, 58.88; $\mathrm{H}, 3.92 ; \mathrm{N}, 4.30 \%$.

Di-tert-butyl 2- (6-(1,3-dimethyl-2,4,6-trioxo-hexahydropyrimidin-5-yl)phenanthridine5(6H)-yl)fumarate $4 \mathrm{~h}$. Pink powder, yield $96 \%, 0.54 \mathrm{~g} \mathrm{mp} \mathrm{135-137}{ }^{\circ} \mathrm{C}, \mathrm{IR}\left(v_{\max }, \mathrm{cm}^{-1}\right): 1681$ and $1740(\mathrm{C}=\mathrm{O}) .{ }^{1} \mathrm{H}$ NMR $\left(500.1 \mathrm{MHz}, \mathrm{CDCl}_{3}\right), \delta_{\mathrm{H}} 1.30$ and $1.57\left(18 \mathrm{H}, 2 \mathrm{~s}, 2 \mathrm{OC}\left(\mathrm{CH}_{3}\right)_{3}\right), 2.73$ and $3.38\left(6 \mathrm{H}, 2 \mathrm{~s}, 2 \mathrm{NCH}_{3}\right), 4.69\left(1 \mathrm{H}, \mathrm{d},{ }^{3} J_{\mathrm{HH}}=7.4 \mathrm{~Hz}, \mathrm{NCHCH}\right), 5.01\left(1 \mathrm{H}, \mathrm{d},{ }^{3} J_{\mathrm{HH}}=7.4 \mathrm{~Hz}\right.$, $\mathrm{O}=\mathrm{C}-\mathrm{C} H-\mathrm{C}=\mathrm{O}), 5.64 \quad\left(1 \mathrm{H}, \mathrm{s}, \mathrm{N}-\mathrm{C}=\mathrm{C} H-\mathrm{CO}_{2} \mathrm{C}\left(\mathrm{CH}_{3}\right)_{3}\right), 6.66-7.80\left(8 \mathrm{H}_{\text {aro }}, \mathrm{m}, 8 \mathrm{CH}\right) \cdot{ }^{13} \mathrm{C}$ NMR $\left(125.8 \mathrm{MHz}, \mathrm{CDCl}_{3}\right) \delta_{\mathrm{C}} 26.71$ and $26.99\left(6 \mathrm{C}, 2 \mathrm{CO}_{2} \mathrm{C}\left(\mathrm{CH}_{3}\right)_{3}\right), 27.02$ and $27.94\left(2 \mathrm{C}, 2 \mathrm{NCH}_{3}\right)$, $65.79(1 \mathrm{C}, \mathrm{NCHCH}), 67.05(1 \mathrm{C}, \mathrm{O}=\mathrm{C}-\mathrm{CH}-\mathrm{C}=\mathrm{O}), 73.20\left(1 \mathrm{C}, \mathrm{N}-\mathrm{C}=\mathrm{CH}-\mathrm{CO}_{2} \mathrm{C}\left(\mathrm{CH}_{3}\right)_{3}\right), 81.62$ and $81.78\left(2 \mathrm{C}, 2 \mathrm{CO} 2 \mathrm{C}\left(\mathrm{CH}_{3}\right)_{3}\right), 111.65,116.09,118.11,121.00$ and 121.06 (6C, phenanthridine), $124.14\left(1 \mathrm{C}, \mathrm{N}-\mathrm{C}=\mathrm{CH}-\mathrm{CO}_{2} \mathrm{C}\left(\mathrm{CH}_{3}\right)_{3}\right), 124.39,125.99,128.79,129.20,129.67$ and $143.55(6 \mathrm{C}$, phenanthridine), 149.49 (1C, N-CO-N), 166.45 and 167.04 (2C, 2C-CO-C), 167.21 and 169.93 (2C, $2 \mathrm{C}=\mathrm{O}$, ester). Anal. Calcd for $\mathrm{C}_{31} \mathrm{H}_{35} \mathrm{~N}_{3} \mathrm{O}_{7}$ (561.27): $\mathrm{C}, 66.28 ; \mathrm{H}, 6.28 ; \mathrm{N}, 7.48 \%$, Found: $\mathrm{C}$, $65.96 ; \mathrm{H}, 6.37 ; \mathrm{N}, 7.58 \%$.

Dimethyl 2-(1-(2,4-dioxopentan-3-yl)isoquinolin-2(1H)-yl)maleate 8i. Brown powder, yield

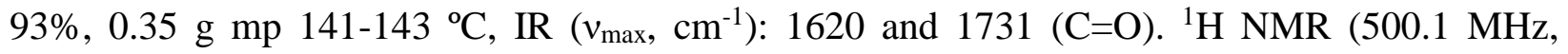
$\left.\mathrm{CDCl}_{3}\right), \delta \mathrm{H} 1.79$ and $2.23\left(6 \mathrm{H}, 2 \mathrm{~s}, 2 \mathrm{CH}_{3}\right), 3.60$ and $3.80\left(6 \mathrm{H}, 2 \mathrm{~s}, 2 \mathrm{OCH}_{3}\right), 3.90\left(1 \mathrm{H}, \mathrm{d},{ }^{3} \mathrm{~J}_{\mathrm{HH}}=\right.$ $10.3 \mathrm{~Hz}, \mathrm{O}=\mathrm{C}-\mathrm{CH}-\mathrm{C}=\mathrm{O}), 5.24\left(1 \mathrm{H}, \mathrm{s}, \mathrm{N}-\mathrm{C}=\mathrm{CH}-\mathrm{CO}_{2} \mathrm{CH}_{3}\right), 5.54\left(1 \mathrm{H}, \mathrm{d},{ }^{3} J_{\mathrm{HH}}=10.3 \mathrm{~Hz}\right.$, $\mathrm{NCHCH}), 6.12\left(1 \mathrm{H}, \mathrm{d},{ }^{3} J_{\mathrm{HH}}=7.6 \mathrm{~Hz}, \mathrm{~N}-\mathrm{CH}=\mathrm{CH}\right.$, isoquinoline $), 6.61\left(1 \mathrm{H}, \mathrm{d},{ }^{3} J_{\mathrm{HH}}=7.6 \mathrm{~Hz}, \mathrm{~N}-\right.$ $\mathrm{CH}=\mathrm{CH}$, isoquinoline), 7.04-7.40 (4Haro, $\mathrm{m}, 4 \mathrm{CH}$, isoquinoline), ${ }^{13} \mathrm{C} \mathrm{NMR}\left(125.8 \mathrm{MHz}, \mathrm{CDCl}_{3}\right)$ $\delta_{\mathrm{C}} 51.79$ and $53.73\left(2 \mathrm{OCH}_{3}\right), 59.41(\mathrm{NCHCH}), 69.90(\mathrm{O}=\mathrm{C}-\mathrm{CH}-\mathrm{C}=\mathrm{O}), 95.23(\mathrm{~N}-\mathrm{C}=\mathrm{CH}-$ $\left.\mathrm{CO}_{2} \mathrm{CH}_{3}\right), 123.14\left(\mathrm{~N}-\mathrm{C}=\mathrm{CH}-\mathrm{CO}_{2} \mathrm{CH}_{3}\right), 124.78,125.70,126.17,127.22,127.94,128.22,128.76$ and 130.11 ( $8 \mathrm{C}$, isoquinoline), 163.90 and $165.13(2 \mathrm{C}=\mathrm{O}$, ester), 198.82 and $199.61(2 \mathrm{C}=\mathrm{O})$. MS, $\mathrm{m} / \mathrm{z}(\%)=228\left(\mathrm{C}_{14} \mathrm{H}_{14} \mathrm{NO}_{2}, 66\right), 208\left(\mathrm{C}_{13} \mathrm{H}_{6} \mathrm{NO}_{2}, 47\right), 143\left(\mathrm{C}_{6} \mathrm{H}_{7} \mathrm{O}_{4}, 14\right), 129\left(\mathrm{C}_{9} \mathrm{H}_{7} \mathrm{~N}, 46\right)$, $99\left(\mathrm{C}_{5} \mathrm{H}_{7} \mathrm{O}_{2}, 10\right), 59\left(\mathrm{CO}_{2} \mathrm{CH}_{3}, 22\right)$. Anal. Calcd for $\mathrm{C}_{20} \mathrm{H}_{21} \mathrm{NO}_{6}$ (371.16): C, 64.66; H, 5.70; N, $3.77 \%$, Found: C, 64.71; H, 5.63; N, 3.81\%.

Dimethyl 2-(1-(1,3-dioxo-1,3-diphenylpropan-2-yl)isoquinolin-2(1H)-yl)maleate 8j. Brown powder, yield $91 \%$, 0.45g mp 156-158 ${ }^{\circ} \mathrm{C}$, IR $\left(v_{\max }, \mathrm{cm}^{-1}\right): 1618$ and $1736(\mathrm{C}=\mathrm{O}) .{ }^{1} \mathrm{H}$ NMR $\left(500.1 \mathrm{MHz}, \mathrm{CDCl}_{3}\right), \delta \mathrm{H} 3.62$ and $3.78\left(6 \mathrm{H}, 2 \mathrm{~s}, 2 \mathrm{OCH}_{3}\right), 5.75\left(1 \mathrm{H}, \mathrm{d},{ }^{3} J_{\mathrm{HH}}=9.9 \mathrm{~Hz}, \mathrm{O}=\mathrm{C}-\mathrm{CH}-\right.$ $\mathrm{C}=\mathrm{O}), 5.86\left(1 \mathrm{H}, \mathrm{s}, \mathrm{N}-\mathrm{C}=\mathrm{CH}-\mathrm{CO}_{2} \mathrm{CH}_{3}\right), 6.03\left(1 \mathrm{H}, \mathrm{d},{ }^{3} J_{\mathrm{HH}}=9.9 \mathrm{~Hz}, \mathrm{NCHCH}\right), 6.19\left(1 \mathrm{H}, \mathrm{d},{ }^{3} J_{\mathrm{HH}}=\right.$ $7.5 \mathrm{~Hz}, \mathrm{~N}-\mathrm{CH}=\mathrm{CH}$, isoquinoline), $6.56\left(1 \mathrm{H}, \mathrm{d},{ }^{3} J_{\mathrm{HH}}=7.5 \mathrm{~Hz}, \mathrm{~N}-\mathrm{CH}=\mathrm{CH}\right.$, isoquinoline), 7.10$8.45\left(14 \mathrm{H}_{\text {aro }}, \mathrm{m}, 14 \mathrm{CH}\right.$, isoquinoline and $\left.2 \mathrm{C}_{6} \mathrm{H}_{5}\right),{ }^{13} \mathrm{C} \mathrm{NMR}\left(125.8 \mathrm{MHz}, \mathrm{CDCl}_{3}\right) \delta_{\mathrm{C}} 51.27$ and 
$52.81\left(2 \mathrm{OCH}_{3}\right), 58.32(\mathrm{NCHCH}), 63.12(\mathrm{O}=\mathrm{C}-\mathrm{CH}-\mathrm{C}=\mathrm{O}), 97.11\left(\mathrm{~N}-\mathrm{C}=\mathrm{CH}-\mathrm{CO}_{2} \mathrm{CH}_{3}\right), 124.09(\mathrm{~N}-$ $\left.C=\mathrm{CH}-\mathrm{CO}_{2} \mathrm{CH}_{3}\right), 125.90,126.70$ and126.84, (3C, isoquinoline), $127.16\left(1 \mathrm{C}, 2 \mathrm{C}_{6} \mathrm{H}_{5}\right), 128.22$ and 128.67 (2C, isoquinoline), 128.80 and $128.84\left(2 \mathrm{C}, 2 \mathrm{C}_{6} \mathrm{H}_{5}\right), 128.76$ and 128. $89(2 \mathrm{C}$, isoquinoline), $129.17,129.94$ and $130.28\left(3 \mathrm{C}, 3 \mathrm{C}_{6} \mathrm{H}_{5}\right), 130.64(1 \mathrm{C}$, isoquinoline), 164.75 and $166.80\left(2 \mathrm{C}=\mathrm{O}\right.$, ester), 191.81 and $191.90(2 \mathrm{C}=\mathrm{O}) . \mathrm{MS}, \mathrm{m} / \mathrm{z} \%=180\left(\mathrm{C}_{12} \mathrm{H}_{6} \mathrm{NO}, 12\right), 143$ $\left(\mathrm{C}_{6} \mathrm{H}_{7} \mathrm{O}_{4}, 54\right), 129\left(\mathrm{C}_{9} \mathrm{H}_{7} \mathrm{~N}, 100\right), 128\left(\mathrm{C}_{5} \mathrm{H}_{4} \mathrm{O}_{4}, 29\right), 102\left(\mathrm{C}_{7} \mathrm{H}_{2} \mathrm{O}, 51\right), 97\left(\mathrm{C}_{4} \mathrm{HO}_{3}, 9\right), 76\left(\mathrm{C}_{6} \mathrm{H}_{4}\right.$, 15), $59\left(\mathrm{CO}_{2} \mathrm{CH}_{3}, 27\right)$. Anal. Calcd for $\mathrm{C}_{30} \mathrm{H}_{25} \mathrm{NO}_{6}$ (495.20): C, 72.70; H, 5.09; N, $2.83 \%$, Found: C, 72.85; H, 4.94; N, $2.97 \%$.

\section{Acknowledgements}

We gratefully acknowledge financial support from the Research Council of University of Sistan and Balouchestan.

\section{References}

1. Laszlo, P. Organic Reactions: Simplisity and Logic; Wiley: New York, 1995.

2. Xie, C.; Zhang, Y.; Huang, Z.; Xu, P. J. Org. Chem. 2007, 72, 5431.

3. Ishikawa, T. Med. Res. Rev. 2001, 21, 61.

4. Nakanishi, T.; Suzuki, M.; Siamoto, A.; Kabasawa, T. J. Nat. Prod. 1999, 621, 864.

5. Sripada, L.; Teske, J. A.; Deiters, A. Org. Biomol. Chem. 2008, 6, 263.

6. Phillips, S. D.; Castle, R. N. J. Heterocycl. Chem. 1981, 18, 223.

7. Denny, W. A. Curr. Med. Chem. 2002, 9, 1655.

8. Lynch, M. A.; Duval, O.; Sukhanova, A.; Devy, J.; Mackay, S. P.; Waigh, R. D.; Nabiev, I. Bioorg. Med. Chem. Lett. 2001, 11, 2643.

9. Janin, Y. L.; Croisy, A.; Riou, J. F.; Bisagni, E. J. Med. Chem. 1993, 36, 3686.

10. Atwell, G. L.; Baguley, B. C.; Denny, W. A. J. Med. Chem. 1998, 31, 774.

11. Luedtke, N. W.; Liu, Q.; Tor, Y. Bioorg. Med. Chem. 2003, 11, 5235.

12. Nishiwaki, H.; Miura, M.; Imai, K.; Ohno, R.; Kawashima, K. Cancer. Res. 1974, 34, 2699.

13. Guntaka, R. V.; Mahy, B. W.; Bishop, J. M.; Varmus, H. E. Nature. 1975, 253, 507.

14. Nakanishi, T.; Suzuki, M.; Mashiba, A.; Ishikawa, K.; Yokotsuka, T. J. Org. Chem. 1998, $63,4235$.

15. Stermitz, F. R.; Larson, K. A.; Kim, D. K. J. Med. Chem. 1973, 16, 939.

16. Osornio, Y. M.; Miranda, L. D. Rev. Soc. Quim. Mex. 2004, 48, 288.

17. Pettit, G. R.; Gaddamidi, V.; Goswami, A.; Cragg, G. M. J. Nat. Prod. 1984, 47, 796.

18. 18.Harayama, T.; Akamatsu, H.; Okamura, K.; Miyagoe, T.; Akiyama, T.; Abe, H.; Takeuchi, Y. J. Chem. Soc., Perkin Trans. 1 2001, 523.

19. Vavreckova, C.; Gawlik, I.; Muller, K. Planta. Med. 1996, 62, 397. 
20. Schmeller, T.; Latz-Bruning, B.; Wink, M. Phytochemistry 1997, 44, 257.

21. 21.Vrba, J.; Hrbac, J.; Ulrichova, J.; Modriansky, M. Chemico-Biological Interaction 2004, 147,35 .

22. 22. Whittaker, J.; Mcfadyen, W. D.; Baguley, B. C.; Murray, V. Anticancer Drug Des. 2001, $16,81$.

23. 23. Alba, F. J.; Bermudez, A.; Daban, J. R. Electrophoresis 2001, 22, 399.

24. 24. Mullins, S. T.; Sammes, P. G.; West, R. M. Yahioglu, G. J. Chem. Soc., Perkin Trans. 1 1996, 75.

25. 25. Ewing, J.; Hughes, G.; Ritchie, E.; Taylor, W. C. Nature 1952, 169, 618.

26. 26. Yadav, J. S.; Subba Reddy, B. V.; Yadav, N. N.; Gupta, M. K. Tetrahedron Lett. 2008, 49, 2815.

27. 27. Yavari, I.; Ghazanfarpour-Darijani, M.; Sabbaghan, M.; Hossaini, Z. Tetrahedron Lett. 2007, 48, 3749.

28. Hadda, T. B.; Rahima, B.; Kerbal, A.; Bennani, B.; Al Houari, G.; Baba, B. F.; Akkurt, M.; Demailly, G.; Benazza, M. Arkivoc. 2008, (ii), 1.

29. Orito, K.; Harada, R.; Uchiito, S.; Tokuda, M. Org. Lett. 2000, 2, 1799.

30. Yavari, I.; Hossaini, Z.; Sabbaghan, M. Ghazanfarpour-Darijani, M. Monatsh. Chem. 2007, $138,677$.

31. Alizadeh, A.; Zohreh, N. Synthesis 2008, 429.

32. Terzidis, M. A.; Tsoleridis, C. A.; Stephanidou-Stephanatou, J. Synlett 2009, 2, 229.

33. Diaz, J. L.; Miguel. M.; Lavilla, R. J. Org. Chem. 2004, 69, 3550.

34. Nassiri, M.; Maghsoodlou, M. T.; Heydari, R.; Habibi-Khorassani, S. M. Mol. Divers. 2008, $12,111$.

35. Anderson. W. K.; Heider, A. R.; Raju, N.; Yucht, J. A. J. Med. Chem. 1988, 31, 2097.

36. Goldbrunner, M.; Loidl, G.; Polossek, T.; Mannschreck, A.; Von Angerer, E. J. Med. Chem. 1997, 40, 3524.

37. Ambros, R.; Von Angerer, S.; Wiegrebe, W. Arch. Pharm. 1988, 321, 481.

38. Acheson, R. M.; Elmore, N. F. Adv. Heterocycl. Chem. 1978, 23, 263.

39. Kalantari, M.; Islami, M. R.; Hasani, Z.; Saidi, K. Arkivoc 2006, (x), 55.

40. Islami, M. R.; Mollazehi, F.; Sheibani, H. Arkivoc 2005, (x), 25.

41. Hassani, Z.; Islami, M. R.; Sheibani, H.; Kalantari, M.; Saidi, K. Arkivoc 2006, (i), 89.

42. Maghsoodlou, M. T.; Habibi-Khorassani, S. M.; Rofouei, M. K.; Adhamdoust, S. R.; Nassiri, M. Arkivoc 2006, (xii), 138.

43. Nair, V.; Pattoorpadi Babu, B.; Varghese, V.; Sinu, C. R.; Rajan Paul, R.; Anabha, E. R.; Suresh, E. Tetrahedron Lett. 2009, 50, 3716.

44. Nair, V.; Ramachandran Sreekanth, A.; Pillai Abhilash, N.; Thankappan Nair Biju, A.; Varma, L.; Viji, S, Mathew, S. Arkivoc 2005, (xi), 178.

45. Terzidis, M. A.; Tsoleridis, C. A.; Stephanidou-Stephanatou, J. Synlett 2009, 2, 229.

46. Kazemian, M. A.; Nassiri, M.; Maghsoodlou, M. T.; Habibi-Khorassani, S. M.; VasheghaniFarahani, F. Arkivoc 2008, (xvii), 173. 
47. Eliel, E.; Wilen, S. H. Stereochemistry of Organic Compounds. Wiley: New York, p 570.

48. Yavari, I.; Nasiri, F.; Djahaniani, H. Phosphorus, Sulfur and Silicon 2003, 178, 2627.

49. Silverstein, R. M.; Webester, F. X.; Kiemle, D. J. Spectrometric Identification of Organic Compounds. John Wiley and Sons, Editor, Rebbic Brennan, pp 141-143, 2006, Seventh Edition. 